Acta Crystallographica Section E

Structure Reports

Online

ISSN 1600-5368

\section{Andrei S. Batsanov* and Igor F. Perepichka}

Department of Chemistry, University of Durham, South Road, Durham DH1 3LE, England

Correspondence e-mail:

a.s.batsanov@durham.ac.uk

\section{Key indicators}

Single-crystal $\mathrm{X}$-ray study

$T=150 \mathrm{~K}$

Mean $\sigma(\mathrm{C}-\mathrm{C})=0.007 \AA$

$R$ factor $=0.041$

$w R$ factor $=0.106$

Data-to-parameter ratio $=9.8$

For details of how these key indicators were automatically derived from the article, see http://journals.iucr.org/e.

\title{
9-Dicyanomethylene-4,5-dinitrofluorene- 2,7-disulfonamide
}

The title compound, $\mathrm{C}_{16} \mathrm{H}_{8} \mathrm{~N}_{6} \mathrm{O}_{8} \mathrm{~S}_{2}$, has a twisted fluorene moiety due to steric repulsion between the 4- and 5-nitro groups.

\section{Comment}

2,4,5,7-Tetranitro-9-dicyanomethylenefluorene (DTeF) (Silverman et al., 1974) is a strong electron acceptor, used as a component of charge-transfer complexes (CTC) (Perepichka et al., 1998; Batsanov, Bryce et al., 2001; Batsanov, Perepichka et al., 2001). We also prepared and structurally characterized similar complexes with 2,7-bis( $n$-butylsulfonyl)- and 2,7-bis(phenylsulfonyl)-9-dicyanomethylene-4,5-dinitrofluorene (Perepichka et al., 2000). The title compound, (I), was prepared in the course of the same study.<smiles>N#CC(C#N)=C1c2cc(S(N)(=O)=O)cc([N+](=O)[O-])c2-c2c1cc(S(N)(=O)=O)cc2[N+](=O)[O-]</smiles>

(I)

The fluorene system of (I), like that of DTeF, adopts a twisted conformation (Fig. 1), due to steric repulsion between the nitro groups in positions 4 and 5 [intramolecular contacts

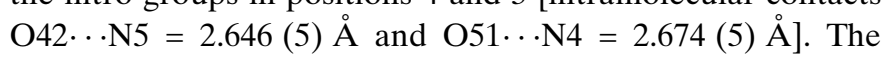
strain is relieved by (i) both nitro groups tilting out of the aromatic plane in opposite directions and (ii) the fluorene moiety itself twisting substantially. Thus, the $13 \mathrm{C}$ atoms of the fluorene moiety show an average deviation of $0.13 \AA$ from their mean plane. Both benzene rings adopt 'sofa' conformations, atoms $\mathrm{C} 11$ and $\mathrm{C} 12$ deviating by 0.12 and $0.06 \AA$ from the planes of the essentially planar moieties $\mathrm{C} 1-\mathrm{C} 4 / \mathrm{C} 10$ and $\mathrm{C} 5-\mathrm{C} 8 / \mathrm{C} 13$, respectively. The latter planes form a dihedral angle of $15.2(3)^{\circ}$. The twist around the $\mathrm{C} 9=\mathrm{C} 14$ bond, i.e. the dihedral angle between the $\mathrm{C} 9 / \mathrm{C} 10 / \mathrm{C} 13 / \mathrm{C} 14$ and $\mathrm{C} 9 / \mathrm{C} 14 / \mathrm{C} 15 /$ $\mathrm{C} 16 / \mathrm{N} 15 / \mathrm{N} 16$ planes, is $11.5(3)^{\circ}$. The bond lengths and angles in (I) (Table 1) are consistent with those observed in other distorted fluorene systems.

The $\mathrm{NH}_{2}$ groups adopt trans orientations with respect to the mean fluorene plane. All amine $\mathrm{H}$ atoms participate in intermolecular hydrogen bonds (Table 2), although one of these ( $\mathrm{H} 71 \cdots \mathrm{O} 41)$ is very weak.
Received 15 September 2004 Accepted 22 September 2004 Online 30 September 2004 


\section{Experimental}

4,5-Dinitro-9-fluorenone-2,7-disulfonyl dichloride [(II); $200 \mathrm{mg}$, $0.43 \mathrm{~mol}$ ] (Mysyk et al., 1997) was dissolved in dry dioxane $(10 \mathrm{ml})$ and ammonia ( $35 \%$ solution in water, $0.2 \mathrm{ml}$ ) was added dropwise with intense stirring. The mixture was stirred at room temperature for $1 \mathrm{~h}$ and poured into water. The solid was filtered off, washed with warm water, dried and recrystallized from acetone, yielding pale yellow crystals of 4,5-dinitro-9-fluorenone-2,7-disulfonamide, (III) (130 mg, yield 71\%, m.p. $>573 \mathrm{~K}$ ). ${ }^{1} \mathrm{H}$ NMR (400 MHz, acetone- $d_{6}$ ): $\delta$ $8.63(2 \mathrm{H}, d, J=1.5 \mathrm{~Hz}, \mathrm{H}-3,6), 8.50(2 \mathrm{H}, d, J=1.5 \mathrm{~Hz}, \mathrm{H}-1,8), 7.19$ $\left(4 \mathrm{H}, s, \mathrm{SO}_{2} \mathrm{NH}_{2}\right) ;{ }^{13} \mathrm{C}$ NMR $\left(100 \mathrm{MHz}\right.$, acetone- $\left.d_{6}\right): \delta 186.56(\mathrm{C}=\mathrm{O})$, 149.28, 147.15, 139.40, 136.61, 128.86, 126.07. Analysis found: C 36.42 , $\mathrm{H} 1.95, \mathrm{~N} 13.18, \mathrm{~S} 14.89 \% ; \mathrm{C}_{13} \mathrm{H}_{8} \mathrm{~N}_{4} \mathrm{O}_{9} \mathrm{~S}_{2}$ requires: $\mathrm{C} 36.45, \mathrm{H} 1.88, \mathrm{~N}$ 13.08 , S $14.97 \%$. Compound (III) (300 mg, $0.70 \mathrm{mmol}$ ) and malononitrile $(100 \mathrm{mg}, 167 \mathrm{mmol})$ in dimethylformamide $(1.5 \mathrm{ml})$ were stirred at room temperature for $4 \mathrm{~h}$ and diluted with methanol $(5 \mathrm{ml})$, resulting in precipitation. After keeping this solution at $273 \mathrm{~K}$ for $6 \mathrm{~h}$, the solid obtained was filtered off and washed with water, yielding crude product (I) as a yellow-green solid. This was dissolved in a minimal amount of hot acetone and diluted with a fourfold volume of hot methanol. On cooling, a bright yellow solid was collected, washed with methanol and dried, giving $210 \mathrm{mg}$ of (I) (yield 63\%), m.p. $>573 \mathrm{~K} .{ }^{1} \mathrm{H}$ NMR (300 MHz, acetone- $d_{6}+c a 0.2$ drop $\mathrm{CF}_{3} \mathrm{CO}_{2} \mathrm{D}$ ): $\delta$ $9.36(2 \mathrm{H}, d, J=1.4 \mathrm{~Hz}, \mathrm{H}-1.8), 8.71(2 \mathrm{H}, d, J=1.4 \mathrm{~Hz}, \mathrm{H}-3,6), 7.32$ $\left(4 \mathrm{H}, s, \mathrm{SO}_{2} \mathrm{NH}_{2}\right)$. Analysis found: $\mathrm{C} 40.22, \mathrm{H} 1.65, \mathrm{~N} 17.78, \mathrm{~S} 13.37$; $\mathrm{C}_{16} \mathrm{H}_{8} \mathrm{~N}_{6} \mathrm{O}_{8} \mathrm{~S}_{2}$ requires: $\mathrm{C} 40.34, \mathrm{H} 1.69, \mathrm{~N} 17.64, \mathrm{~S} 13.46 \%$. Single crystals of (I) of X-ray quality were obtained by slow evaporation (over several days) at room temperature of a solution of (I) (10 mg) in $\mathrm{PhCl}(10 \mathrm{ml})$ and acetonitrile $(3 \mathrm{ml})$.

\section{Crystal data \\ $\mathrm{C}_{16} \mathrm{H}_{8} \mathrm{~N}_{6} \mathrm{O}_{8} \mathrm{~S}_{2}$ \\ $M_{r}=476.40$ \\ Orthorhombic, Pna2 1 \\ $a=9.574(2) \AA$ \\ $b=10.778(6) \AA$ \\ $c=17.461(5) \AA$ \\ $V=1801.8(12) \AA^{3}$ \\ $Z=4$ \\ $D_{x}=1.756 \mathrm{Mg} \mathrm{m}^{-3}$}

\section{Data collection}

Rigaku AFC-6S four-circle diffractometer

$2 \theta / \omega$ scans

Absorption correction: $\psi$ scan

(TEXSAN; Molecular Structure Corporation, 1989)

$T_{\min }=0.745, T_{\max }=0.848$

2381 measured reflections

1942 independent reflections

\section{Refinement}

Refinement on $F^{2}$

$R\left[F^{2}>2 \sigma\left(F^{2}\right)\right]=0.041$

$w R\left(F^{2}\right)=0.106$

$S=1.03$

1942 reflections

199 parameters

$\mathrm{H}$-atom parameters constrained
$\mathrm{Cu} K \alpha$ radiation

Cell parameters from 25

reflections

$\theta=15.5-25.0^{\circ}$

$\mu=3.30 \mathrm{~mm}^{-1}$

$T=150(2) \mathrm{K}$

Needle, yellow

$0.45 \times 0.06 \times 0.05 \mathrm{~mm}$

1730 reflections with $I>2 \sigma(I)$

$R_{\text {int }}=0.033$

$\theta_{\text {max }}=75.1^{\circ}$

$h=-1 \rightarrow 12$

$k=-1 \rightarrow 13$

$l=-1 \rightarrow 21$

3 standard reflections

every 147 reflections

intensity decay: $0.9 \%$

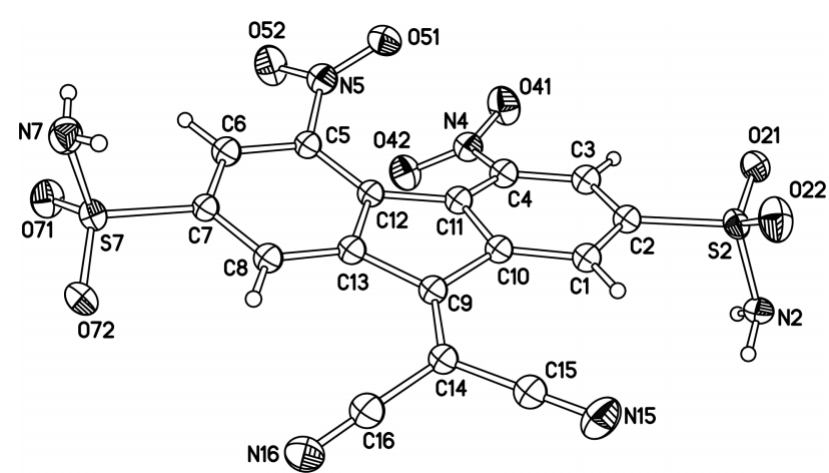

Figure 1

The molecular structure of (I). Displacement ellipsoids are drawn at the $50 \%$ probability level.

Table 1

Selected geometric parameters $\left(\AA,^{\circ}\right)$.

\begin{tabular}{|c|c|c|c|}
\hline $\mathrm{S} 2-\mathrm{O} 22$ & $1.424(4)$ & $\mathrm{C} 6-\mathrm{C} 7$ & $1.384(6)$ \\
\hline $\mathrm{S} 2-\mathrm{O} 21$ & $1.433(4)$ & $\mathrm{C} 7-\mathrm{C} 8$ & $1.394(6)$ \\
\hline $\mathrm{S} 2-\mathrm{N} 2$ & $1.612(4)$ & $\mathrm{C} 8-\mathrm{C} 13$ & $1.387(7)$ \\
\hline $\mathrm{S} 2-\mathrm{C} 2$ & $1.785(5)$ & $\mathrm{C} 9-\mathrm{C} 14$ & $1.358(6)$ \\
\hline $\mathrm{S} 7-\mathrm{O} 72$ & $1.428(4)$ & $\mathrm{C} 9-\mathrm{C} 13$ & $1.469(6)$ \\
\hline $\mathrm{S} 7-\mathrm{O} 71$ & $1.432(3)$ & $\mathrm{C} 9-\mathrm{C} 10$ & $1.475(6)$ \\
\hline $\mathrm{S} 7-\mathrm{N} 7$ & $1.606(4)$ & $\mathrm{C} 10-\mathrm{C} 11$ & $1.410(6)$ \\
\hline $\mathrm{S} 7-\mathrm{C} 7$ & $1.784(5)$ & $\mathrm{C} 11-\mathrm{C} 12$ & $1.473(6)$ \\
\hline $\mathrm{C} 1-\mathrm{C} 2$ & $1.383(7)$ & $\mathrm{C} 12-\mathrm{C} 13$ & $1.429(6)$ \\
\hline $\mathrm{C} 1-\mathrm{C} 10$ & $1.396(6)$ & $\mathrm{C} 14-\mathrm{C} 15$ & $1.435(6)$ \\
\hline $\mathrm{C} 2-\mathrm{C} 3$ & $1.381(7)$ & $\mathrm{C} 14-\mathrm{C} 16$ & $1.446(7)$ \\
\hline $\mathrm{C} 3-\mathrm{C} 4$ & $1.393(6)$ & $\mathrm{C} 15-\mathrm{N} 15$ & $1.144(7)$ \\
\hline $\mathrm{C} 4-\mathrm{C} 11$ & $1.387(7)$ & $\mathrm{C} 16-\mathrm{N} 16$ & $1.146(7)$ \\
\hline $\mathrm{C} 4-\mathrm{N} 4$ & $1.476(6)$ & $\mathrm{N} 4-\mathrm{O} 41$ & $1.218(5)$ \\
\hline $\mathrm{C} 5-\mathrm{C} 12$ & $1.387(6)$ & $\mathrm{N} 4-\mathrm{O} 42$ & $1.236(5)$ \\
\hline $\mathrm{C} 5-\mathrm{C} 6$ & $1.391(6)$ & $\mathrm{N} 5-\mathrm{O} 52$ & $1.219(5)$ \\
\hline $\mathrm{C} 5-\mathrm{N} 5$ & $1.465(6)$ & $\mathrm{N} 5-\mathrm{O} 51$ & $1.239(5)$ \\
\hline $\mathrm{C} 2-\mathrm{C} 1-\mathrm{C} 10$ & $117.7(4)$ & $\mathrm{C} 14-\mathrm{C} 9-\mathrm{C} 10$ & $127.1(4)$ \\
\hline $\mathrm{C} 3-\mathrm{C} 2-\mathrm{C} 1$ & $122.6(4)$ & $\mathrm{C} 13-\mathrm{C} 9-\mathrm{C} 10$ & $106.8(4)$ \\
\hline $\mathrm{C} 3-\mathrm{C} 2-\mathrm{S} 2$ & $117.4(4)$ & $\mathrm{C} 1-\mathrm{C} 10-\mathrm{C} 11$ & $121.5(4)$ \\
\hline $\mathrm{C} 1-\mathrm{C} 2-\mathrm{S} 2$ & $120.0(4)$ & $\mathrm{C} 1-\mathrm{C} 10-\mathrm{C} 9$ & $130.4(4)$ \\
\hline $\mathrm{C} 2-\mathrm{C} 3-\mathrm{C} 4$ & $118.2(5)$ & $\mathrm{C} 11-\mathrm{C} 10-\mathrm{C} 9$ & $108.0(4)$ \\
\hline $\mathrm{C} 11-\mathrm{C} 4-\mathrm{C} 3$ & $121.7(4)$ & $\mathrm{C} 4-\mathrm{C} 11-\mathrm{C} 10$ & $117.4(4)$ \\
\hline $\mathrm{C} 11-\mathrm{C} 4-\mathrm{N} 4$ & $122.2(4)$ & $\mathrm{C} 4-\mathrm{C} 11-\mathrm{C} 12$ & $134.2(4)$ \\
\hline $\mathrm{C} 3-\mathrm{C} 4-\mathrm{N} 4$ & $115.8(4)$ & $\mathrm{C} 10-\mathrm{C} 11-\mathrm{C} 12$ & $108.3(4)$ \\
\hline $\mathrm{C} 12-\mathrm{C} 5-\mathrm{C} 6$ & $122.6(4)$ & $\mathrm{C} 5-\mathrm{C} 12-\mathrm{C} 13$ & $116.8(4)$ \\
\hline $\mathrm{C} 12-\mathrm{C} 5-\mathrm{N} 5$ & $122.7(4)$ & $\mathrm{C} 5-\mathrm{C} 12-\mathrm{C} 11$ & $135.2(4)$ \\
\hline $\mathrm{C} 6-\mathrm{C} 5-\mathrm{N} 5$ & $114.5(4)$ & $\mathrm{C} 13-\mathrm{C} 12-\mathrm{C} 11$ & $107.8(4)$ \\
\hline $\mathrm{C} 7-\mathrm{C} 6-\mathrm{C} 5$ & $118.5(4)$ & $\mathrm{C} 8-\mathrm{C} 13-\mathrm{C} 12$ & $121.7(4)$ \\
\hline $\mathrm{C} 6-\mathrm{C} 7-\mathrm{C} 8$ & $121.9(4)$ & $\mathrm{C} 8-\mathrm{C} 13-\mathrm{C} 9$ & $130.2(4)$ \\
\hline $\mathrm{C} 6-\mathrm{C} 7-\mathrm{S} 7$ & $117.8(3)$ & $\mathrm{C} 12-\mathrm{C} 13-\mathrm{C} 9$ & $107.9(4)$ \\
\hline $\mathrm{C} 8-\mathrm{C} 7-\mathrm{S} 7$ & $120.2(4)$ & $\mathrm{C} 9-\mathrm{C} 14-\mathrm{C} 15$ & $123.4(4)$ \\
\hline $\mathrm{C} 13-\mathrm{C} 8-\mathrm{C} 7$ & $118.3(4)$ & $\mathrm{C} 9-\mathrm{C} 14-\mathrm{C} 16$ & $122.2(4)$ \\
\hline $\mathrm{C} 14-\mathrm{C} 9-\mathrm{C} 13$ & $125.9(4)$ & $\mathrm{C} 15-\mathrm{C} 14-\mathrm{C} 16$ & $114.4(4)$ \\
\hline $\mathrm{C} 1-\mathrm{C} 2-\mathrm{S} 2-\mathrm{N} 2$ & $79.0(4)$ & $\mathrm{C} 8-\mathrm{C} 7-\mathrm{S} 7-\mathrm{N} 7$ & $96.3(4)$ \\
\hline
\end{tabular}

Table 2

Hydrogen-bonding geometry $\left(\AA,^{\circ}\right)$.

\begin{tabular}{lllll}
\hline$D-\mathrm{H} \cdots A$ & $D-\mathrm{H}$ & $\mathrm{H} \cdots A$ & $D \cdots A$ & $D-\mathrm{H} \cdots A$ \\
\hline $\mathrm{N} 2-\mathrm{H} 21 \cdots \mathrm{O} 72^{\mathrm{i}}$ & 0.89 & 2.03 & $2.878(6)$ & 159 \\
$\mathrm{~N} 2-\mathrm{H} 22 \cdots \mathrm{O} 52^{\mathrm{ii}}$ & 0.89 & 2.10 & $2.925(5)$ & 153 \\
$\mathrm{~N} 7-\mathrm{H} 71 \cdots \mathrm{O} 41^{\mathrm{iii}}$ & 0.89 & 2.51 & $3.116(6)$ & 126 \\
$\mathrm{~N} 7-\mathrm{H} 72 \cdots \mathrm{N} 2^{\text {iv }}$ & 0.89 & 2.24 & $3.103(6)$ & 162
\end{tabular}

Symmetry codes: (i) $\frac{3}{2}-x, y-\frac{1}{2}, \frac{1}{2}+z$; (ii) $x, y-1, z$; (iii) $1-x, 2-y, z-\frac{1}{2}$; (iv) $1-x, 1-y, z-\frac{1}{2}$. 
Owing to an insufficient number of observed reflections, only the $\mathrm{S}$, $\mathrm{O}$ and $\mathrm{N}$ atoms were refined with anisotropic displacement parameters, the $\mathrm{C}$ atoms being refined in isotropic approximation. Amine $\mathrm{H}$ atoms were refined in isotropic approximation, then constrained with the same bond direction but idealized $\mathrm{N}-\mathrm{H}$ bond lengths (0.89 ̊). Other $\mathrm{H}$ atoms were treated as riding in idealized positions, with $\mathrm{C}-\mathrm{H}$ bond lengths of $0.95 \AA$ and $U_{\text {iso }}(\mathrm{H})=1.2 U_{\text {eq }}(\mathrm{C})$.

Data collection: MSC/AFC Diffractometer Control Software (Molecular Structure Corporation, 1988); cell refinement: $M S C / A F C$ Diffractometer Control Software; data reduction: TEXSAN (Molecular Structure Corporation, 1989); program(s) used to solve structure: SHELXS97 (Sheldrick, 1997); program(s) used to refine structure: SHELXL97 (Sheldrick, 1997); molecular graphics: SHELXTL (Bruker, 1997); software used to prepare material for publication: SHELXTL.

The authors thank Professor M. R. Bryce for fruitful advice. IFP thanks the Royal Society of Chemistry for an International Author grant.

\section{References}

Batsanov, A. S., Bryce, M. R., Chesney, A., Howard, J. A. K., John, D. E., Moore, A. J., Wood, C. L., Gershtenman, H., Becker, J. Y., Khodorkovsky, V. Y., Ellern, A., Bernstein, J., Perepichka, I. F., Rotello, V., Gray, M. \& Cuello, A. O. (2001). J. Mater. Chem. 10, 2181-2191.

Batsanov, A. S., Perepichka, I. F., Bryce, M. R. \& Howard, J. A. K. (2001). Acta Cryst. C57, 1299-1302.

Bruker (1997). SHELXTL. Version 5.10. Bruker AXS Inc., Madison, Wisconsin, USA.

Flack, H. D. (1983). Acta Cryst. A39, 876-881.

Molecular Structure Corporation (1988). MSC/AFC Diffractometer Control Software. MSC, 3200 Research Forest Drive, The Woodlands, TX 77381, USA.

Molecular Structure Corporation (1989). TEXSAN. Version 5.1. MSC, 3200 Research Forest Drive, The Woodlands, TX 77381, USA.

Mysyk, D. D., Perepichka, I. F. \& Sokolov, N. I. (1997). J. Chem. Soc. Perkin Trans. 2, pp. 537-545.

Perepichka, I. F., Kuz'mina, L. G., Perepichka, D. F., Bryce, M. R., Goldenberg, L. M., Popov, A. F. \& Howard, J. A. K. (1998). J. Org. Chem. 63, 6484-6493. Perepichka, I. F., Popov, A. F., Orekhova, T. V., Bryce, M. R., Andrievskii, A. M., Batsanov, A. S., Howard, J. A. K. \& Sokolov, N. I. (2000). J. Org. Chem. 65, 3053-3063.

Sheldrick, G. M. (1997). SHELXS97 and SHELXL97. University of Göttingen, Germany.

Silverman, J., Yannoni, N. F. \& Krukonis, A. P. (1974). Acta Cryst. B30, 1474 1480 . 


\section{supporting information}

Acta Cryst. (2004). E60, o1892-o1894 [https://doi.org/10.1107/S160053680402361X]

\section{9-Dicyanomethylene-4,5-dinitrofluorene-2,7-disulfonamide}

\section{Andrei S. Batsanov and Igor F. Perepichka}

9-Dicyanomethylene-4,5-dinitrofluorene-2,7-disulfonamide

Crystal data

$\mathrm{C}_{16} \mathrm{H}_{8} \mathrm{~N}_{6} \mathrm{O}_{8} \mathrm{~S}_{2}$

$M_{r}=476.40$

Orthorhombic, Pna2 $_{1}$

$a=9.574(2) \AA$

$b=10.778(6) \AA$

$c=17.461(5) \AA$

$V=1801.8(12) \AA^{3}$

$Z=4$

$F(000)=968$

\section{Data collection}

Rigaku AFC-6S four-circle diffractometer

Radiation source: fine-focus sealed tube

Graphite monochromator

$2 \theta / \omega$ scans

Absorption correction: $\psi$ scan

(TEXSAN; Molecular Structure Corporation, 1989)

$T_{\min }=0.745, T_{\max }=0.848$

2381 measured reflections

\section{Refinement}

Refinement on $F^{2}$

Least-squares matrix: full

$R\left[F^{2}>2 \sigma\left(F^{2}\right)\right]=0.041$

$w R\left(F^{2}\right)=0.106$

$S=1.03$

1942 reflections

199 parameters

1 restraint

Primary atom site location: structure-invariant direct methods

Secondary atom site location: difference Fourier map
$D_{\mathrm{x}}=1.756 \mathrm{Mg} \mathrm{m}^{-3}$

Melting point $>573 \mathrm{~K}$

$\mathrm{Cu} K \alpha$ radiation, $\lambda=1.54178 \AA$

Cell parameters from 25 reflections

$\theta=15.5-25.0^{\circ}$

$\mu=3.30 \mathrm{~mm}^{-1}$

$T=150 \mathrm{~K}$

Needle, yellow

$0.45 \times 0.06 \times 0.05 \mathrm{~mm}$

1942 independent reflections 1730 reflections with $I>2 \sigma(I)$

$R_{\text {int }}=0.033$

$\theta_{\text {max }}=75.1^{\circ}, \theta_{\min }=4.8^{\circ}$

$h=-1 \rightarrow 12$

$k=-1 \rightarrow 13$

$l=-1 \rightarrow 21$

3 standard reflections every 147 reflections intensity decay: $0.9 \%$

Hydrogen site location: inferred from neighbouring sites

$\mathrm{H}$-atom parameters constrained

$w=1 /\left[\sigma^{2}\left(F_{\mathrm{o}}{ }^{2}\right)+(0.06 P)^{2}+0.7842 P\right]$ where $P=\left(F_{\mathrm{o}}^{2}+2 F_{\mathrm{c}}^{2}\right) / 3$

$(\Delta / \sigma)_{\max }<0.001$

$\Delta \rho_{\max }=0.37 \mathrm{e} \AA^{-3}$

$\Delta \rho_{\min }=-0.43$ e $\AA^{-3}$

Absolute structure: Flack (1983), 28 Friedel pairs [CHECK]

Absolute structure parameter: 0.01 (3) 


\section{Special details}

Experimental. Needle-like crystal aligned approximately along the $\varphi$ axis. 118 Friedel pairs has been measured. exptl_absorpt_correction_type psi-scan (TEXSAN; Molecular Structure Corporation, 1989), on $108 \psi$-scans of 3 reflections

Geometry. All e.s.d.'s (except the e.s.d. in the dihedral angle between two 1.s. planes) are estimated using the full covariance matrix. The cell e.s.d.'s are taken into account individually in the estimation of e.s.d.'s in distances, angles and torsion angles; correlations between e.s.d.'s in cell parameters are only used when they are defined by crystal symmetry. An approximate (isotropic) treatment of cell e.s.d.'s is used for estimating e.s.d.'s involving 1.s. planes.

Refinement. Refinement of $F^{2}$ against ALL reflections. The weighted $R$-factor $w R$ and goodness of fit $S$ are based on $F^{2}$, conventional $R$-factors $R$ are based on $F$, with $F$ set to zero for negative $F^{2}$. The threshold expression of $F^{2}>\sigma\left(F^{2}\right)$ is used only for calculating $R$-factors(gt) etc. and is not relevant to the choice of reflections for refinement.

Fractional atomic coordinates and isotropic or equivalent isotropic displacement parameters $\left(\AA^{2}\right)$

\begin{tabular}{|c|c|c|c|c|}
\hline & $x$ & $y$ & $z$ & $U_{\text {iso }} * / U_{\text {eq }}$ \\
\hline $\mathrm{S} 2$ & $0.32267(12)$ & $0.29279(10)$ & $0.30553(7)$ & $0.0227(2)$ \\
\hline S7 & $0.85953(12)$ & $0.95636(10)$ & $-0.00931(7)$ & $0.0214(2)$ \\
\hline $\mathrm{C} 1$ & $0.4962(5)$ & $0.4139(4)$ & $0.2047(3)$ & $0.0215(9)^{*}$ \\
\hline $\mathrm{H} 1$ & 0.5019 & 0.3380 & 0.1773 & $0.026^{*}$ \\
\hline $\mathrm{C} 2$ & $0.4190(5)$ & $0.4233(4)$ & $0.2714(3)$ & $0.0212(9)^{*}$ \\
\hline $\mathrm{C} 3$ & $0.4144(5)$ & $0.5305(4)$ & $0.3147(3)$ & $0.0248(9)^{*}$ \\
\hline $\mathrm{H} 3$ & 0.3639 & 0.5331 & 0.3615 & $0.030 *$ \\
\hline $\mathrm{C} 4$ & $0.4858(5)$ & $0.6344(4)$ & 0.2879 & $0.0242(10)^{*}$ \\
\hline C5 & $0.6182(5)$ & $0.8599(4)$ & $0.1692(3)$ & $0.0207(9)^{*}$ \\
\hline C6 & $0.6941(5)$ & 0.9288 & $0.1162(3)$ & $0.0236(10)^{*}$ \\
\hline H6 & 0.6960 & 1.0168 & 0.1187 & $0.028^{*}$ \\
\hline $\mathrm{C} 7$ & $0.7667(5)$ & $0.8662(4)$ & $0.0596(3)$ & $0.0185(9)^{*}$ \\
\hline $\mathrm{C} 8$ & $0.7648(5)$ & $0.7372(4)$ & $0.0541(3)$ & $0.0213(9)^{*}$ \\
\hline H8 & 0.8123 & 0.6958 & 0.0138 & $0.026^{*}$ \\
\hline C9 & $0.6638(5)$ & $0.5371(4)$ & 0.1149 (3) & $0.0201(9)^{*}$ \\
\hline $\mathrm{C} 10$ & $0.5655(5)$ & $0.5201(4)$ & 0.1790 & $0.0200(9)^{*}$ \\
\hline C11 & $0.5517(5)$ & $0.6348(4)$ & $0.2171(3)$ & $0.0211(9)^{*}$ \\
\hline $\mathrm{C} 12$ & $0.6193(5)$ & $0.7312(4)$ & $0.1702(3)$ & $0.0195(9)^{*}$ \\
\hline $\mathrm{C} 13$ & $0.6921(5)$ & $0.6707(4)$ & 0.1093 & $0.0209(9)^{*}$ \\
\hline $\mathrm{C} 14$ & $0.7247(5)$ & $0.4468(4)$ & 0.0721 & $0.0207(9)^{*}$ \\
\hline C15 & $0.6810(5)$ & $0.3196(4)$ & $0.0736(3)$ & $0.0253(10)^{*}$ \\
\hline C16 & $0.8411(5)$ & $0.4717(5)$ & $0.0216(3)$ & $0.0278(10)^{*}$ \\
\hline N2 & $0.4291(4)$ & $0.1993(4)$ & $0.3479(2)$ & $0.0237(9)$ \\
\hline $\mathrm{H} 21$ & 0.4669 & 0.2361 & 0.3885 & $0.028 *$ \\
\hline $\mathrm{H} 22$ & 0.4937 & 0.1670 & 0.3171 & $0.028 *$ \\
\hline N4 & $0.4993(4)$ & $0.7382(4)$ & $0.3425(2)$ & $0.0272(9)^{*}$ \\
\hline N5 & $0.5253(4)$ & $0.9336(4)$ & $0.2179(2)$ & $0.0276(9)^{*}$ \\
\hline N7 & $0.7527(4)$ & $1.0028(4)$ & $-0.0741(2)$ & $0.0290(9)$ \\
\hline H71 & 0.7014 & 1.0593 & -0.0498 & $0.035^{*}$ \\
\hline H72 & 0.7186 & 0.9356 & -0.0971 & $0.035^{*}$ \\
\hline N15 & $0.6447(5)$ & 0.2185 & 0.0715 & $0.0365(11)$ \\
\hline N16 & $0.9357(5)$ & $0.4914(4)$ & $-0.0167(3)$ & $0.0386(11)$ \\
\hline $\mathrm{O} 21$ & $0.2300(4)$ & $0.3387(3)$ & $0.3634(2)$ & $0.0324(8)$ \\
\hline
\end{tabular}




\begin{tabular}{|c|c|c|c|c|}
\hline $\mathrm{O} 22$ & $0.2694(4)$ & $0.2294(4)$ & $0.2402(2)$ & $0.0374(9)$ \\
\hline $\mathrm{O} 41$ & $0.4083(4)$ & $0.7526(4)$ & $0.3904(2)$ & $0.0384(9)$ \\
\hline $\mathrm{O} 42$ & $0.6069(4)$ & $0.8012(3)$ & $0.3374(2)$ & $0.0328(8)$ \\
\hline O51 & $0.4081(4)$ & $0.8909(3)$ & $0.2326(2)$ & $0.0309(8)$ \\
\hline O52 & $0.5671(4)$ & $1.0355(3)$ & 0.2378 (2) & $0.0405(10)$ \\
\hline O71 & $0.9091(4)$ & $1.0654(3)$ & $0.0289(2)$ & $0.0296(8)$ \\
\hline $\mathrm{O} 72$ & $0.9576(4)$ & $0.8737(3)$ & $-0.0438(2)$ & $0.0313(8)$ \\
\hline
\end{tabular}

Atomic displacement parameters $\left(\AA^{2}\right)$

\begin{tabular}{lllllll}
\hline & $U^{11}$ & $U^{22}$ & $U^{33}$ & $U^{12}$ & $U^{13}$ & $U^{23}$ \\
\hline S2 & $0.0195(5)$ & $0.0265(5)$ & $0.0222(5)$ & $-0.0038(4)$ & $-0.0003(5)$ & $0.0048(5)$ \\
S7 & $0.0202(5)$ & $0.0226(5)$ & $0.0213(5)$ & $-0.0031(4)$ & $0.0013(5)$ & $-0.0003(5)$ \\
N2 & $0.020(2)$ & $0.0255(18)$ & $0.025(2)$ & $0.0030(15)$ & $-0.0008(17)$ & $0.0011(16)$ \\
N7 & $0.029(2)$ & $0.032(2)$ & $0.026(2)$ & $-0.0028(17)$ & $-0.0022(19)$ & $0.0052(18)$ \\
N15 & $0.050(3)$ & $0.024(2)$ & $0.036(3)$ & $0.002(2)$ & $0.002(2)$ & $-0.0027(18)$ \\
N16 & $0.037(3)$ & $0.033(2)$ & $0.046(3)$ & $0.0059(19)$ & $0.016(2)$ & $-0.002(2)$ \\
O21 & $0.0257(18)$ & $0.0314(18)$ & $0.0402(19)$ & $0.0000(15)$ & $0.0115(18)$ & $0.0068(16)$ \\
O22 & $0.041(2)$ & $0.042(2)$ & $0.0292(19)$ & $-0.0135(18)$ & $-0.0079(18)$ & $0.0041(17)$ \\
O41 & $0.042(2)$ & $0.041(2)$ & $0.0321(19)$ & $-0.0055(18)$ & $0.0155(18)$ & $-0.0097(18)$ \\
O42 & $0.033(2)$ & $0.0359(18)$ & $0.0297(18)$ & $-0.0071(15)$ & $-0.0014(17)$ & $-0.0050(17)$ \\
O51 & $0.0259(18)$ & $0.0340(18)$ & $0.0327(19)$ & $0.0028(15)$ & $0.0033(16)$ & $-0.0009(16)$ \\
O52 & $0.045(2)$ & $0.0264(18)$ & $0.050(2)$ & $-0.0054(17)$ & $0.020(2)$ & $-0.0156(18)$ \\
O71 & $0.034(2)$ & $0.0239(16)$ & $0.0305(18)$ & $-0.0077(15)$ & $-0.0018(17)$ & $-0.0069(15)$ \\
O72 & $0.0233(17)$ & $0.0338(19)$ & $0.0368(19)$ & $0.0021(15)$ & $0.0099(18)$ & $0.0034(16)$ \\
\end{tabular}

Geometric parameters $\left(A,{ }^{o}\right)$

\begin{tabular}{llll}
\hline $\mathrm{S} 2-\mathrm{O} 22$ & $1.424(4)$ & $\mathrm{C} 7-\mathrm{C} 8$ & $1.394(6)$ \\
$\mathrm{S} 2-\mathrm{O} 21$ & $1.433(4)$ & $\mathrm{C} 8-\mathrm{C} 13$ & $1.387(7)$ \\
$\mathrm{S} 2-\mathrm{N} 2$ & $1.612(4)$ & $\mathrm{C} 8-\mathrm{H} 8$ & 0.9500 \\
$\mathrm{~S} 2-\mathrm{C} 2$ & $1.785(5)$ & $\mathrm{C} 9-\mathrm{C} 14$ & $1.358(6)$ \\
$\mathrm{S} 7-\mathrm{O} 72$ & $1.428(4)$ & $\mathrm{C} 9-\mathrm{C} 13$ & $1.469(6)$ \\
$\mathrm{S} 7-\mathrm{O} 71$ & $1.432(3)$ & $\mathrm{C} 9-\mathrm{C} 10$ & $1.475(6)$ \\
$\mathrm{S} 7-\mathrm{N} 7$ & $1.606(4)$ & $\mathrm{C} 10-\mathrm{C} 11$ & $1.410(6)$ \\
$\mathrm{S} 7-\mathrm{C} 7$ & $1.784(5)$ & $\mathrm{C} 11-\mathrm{C} 12$ & $1.473(6)$ \\
$\mathrm{C} 1-\mathrm{C} 2$ & $1.383(7)$ & $\mathrm{C} 12-\mathrm{C} 13$ & $1.429(6)$ \\
$\mathrm{C} 1-\mathrm{C} 10$ & $1.396(6)$ & $\mathrm{C} 14-\mathrm{C} 15$ & $1.435(6)$ \\
$\mathrm{C} 1-\mathrm{H} 1$ & 0.9500 & $\mathrm{C} 14-\mathrm{C} 16$ & $1.446(7)$ \\
$\mathrm{C} 2-\mathrm{C} 3$ & $1.381(7)$ & $\mathrm{C} 15-\mathrm{N} 15$ & $1.144(7)$ \\
$\mathrm{C} 3-\mathrm{C} 4$ & $1.393(6)$ & $\mathrm{C} 16-\mathrm{N} 16$ & $1.146(7)$ \\
$\mathrm{C} 3-\mathrm{H} 3$ & 0.9499 & $\mathrm{~N} 2-\mathrm{H} 21$ & 0.8901 \\
$\mathrm{C} 4-\mathrm{C} 11$ & $1.387(7)$ & $\mathrm{N} 2-\mathrm{H} 22$ & $1.218(5)$ \\
$\mathrm{C} 4-\mathrm{N} 4$ & $\mathrm{~N} 4-\mathrm{O} 41$ & $1.236(5)$ \\
$\mathrm{C} 5-\mathrm{C} 12$ & $1.476(6)$ & $\mathrm{N} 4-\mathrm{O} 42$ & $1.219(5)$ \\
$\mathrm{C} 5-\mathrm{C} 6$ & $1.387(6)$ & $\mathrm{N} 5-\mathrm{O} 52$ & $1.239(5)$ \\
$\mathrm{C} 5-\mathrm{N} 5$ & $1.391(6)$ & $\mathrm{N} 5-\mathrm{O} 51$ & 0.8901 \\
$\mathrm{C} 6-\mathrm{C} 7$ & $1.465(6)$ & $\mathrm{N} 7-\mathrm{H} 71$ &
\end{tabular}




\begin{tabular}{|c|c|c|c|}
\hline $\mathrm{C} 6-\mathrm{H} 6$ & 0.9500 & $\mathrm{~N} 7-\mathrm{H} 72$ & 0.8900 \\
\hline $\mathrm{O} 22-\mathrm{S} 2-\mathrm{O} 21$ & $120.6(3)$ & $\mathrm{C} 13-\mathrm{C} 8-\mathrm{H} 8$ & 120.8 \\
\hline $\mathrm{O} 22-\mathrm{S} 2-\mathrm{N} 2$ & $107.1(2)$ & $\mathrm{C} 7-\mathrm{C} 8-\mathrm{H} 8$ & 120.9 \\
\hline $\mathrm{O} 21-\mathrm{S} 2-\mathrm{N} 2$ & $106.5(2)$ & $\mathrm{C} 14-\mathrm{C} 9-\mathrm{C} 13$ & $125.9(4)$ \\
\hline $\mathrm{O} 22-\mathrm{S} 2-\mathrm{C} 2$ & $107.2(2)$ & $\mathrm{C} 14-\mathrm{C} 9-\mathrm{C} 10$ & $127.1(4)$ \\
\hline $\mathrm{O} 21-\mathrm{S} 2-\mathrm{C} 2$ & $106.5(2)$ & $\mathrm{C} 13-\mathrm{C} 9-\mathrm{C} 10$ & $106.8(4)$ \\
\hline $\mathrm{N} 2-\mathrm{S} 2-\mathrm{C} 2$ & $108.6(2)$ & $\mathrm{C} 1-\mathrm{C} 10-\mathrm{C} 11$ & $121.5(4)$ \\
\hline $\mathrm{O} 72-\mathrm{S} 7-\mathrm{O} 71$ & $119.4(2)$ & $\mathrm{C} 1-\mathrm{C} 10-\mathrm{C} 9$ & $130.4(4)$ \\
\hline $\mathrm{O} 72-\mathrm{S} 7-\mathrm{N} 7$ & $108.4(2)$ & $\mathrm{C} 11-\mathrm{C} 10-\mathrm{C} 9$ & $108.0(4)$ \\
\hline $\mathrm{O} 71-\mathrm{S} 7-\mathrm{N} 7$ & $106.5(2)$ & $\mathrm{C} 4-\mathrm{C} 11-\mathrm{C} 10$ & $117.4(4)$ \\
\hline $\mathrm{O} 72-\mathrm{S} 7-\mathrm{C} 7$ & $105.8(2)$ & $\mathrm{C} 4-\mathrm{C} 11-\mathrm{C} 12$ & $134.2(4)$ \\
\hline $\mathrm{O} 71-\mathrm{S} 7-\mathrm{C} 7$ & $107.3(2)$ & $\mathrm{C} 10-\mathrm{C} 11-\mathrm{C} 12$ & $108.3(4)$ \\
\hline $\mathrm{N} 7-\mathrm{S} 7-\mathrm{C} 7$ & $109.1(2)$ & $\mathrm{C} 5-\mathrm{C} 12-\mathrm{C} 13$ & $116.8(4)$ \\
\hline $\mathrm{C} 2-\mathrm{C} 1-\mathrm{C} 10$ & $117.7(4)$ & $\mathrm{C} 5-\mathrm{C} 12-\mathrm{C} 11$ & $135.2(4)$ \\
\hline $\mathrm{C} 2-\mathrm{C} 1-\mathrm{H} 1$ & 121.2 & $\mathrm{C} 13-\mathrm{C} 12-\mathrm{C} 11$ & $107.8(4)$ \\
\hline $\mathrm{C} 10-\mathrm{C} 1-\mathrm{H} 1$ & 121.2 & $\mathrm{C} 8-\mathrm{C} 13-\mathrm{C} 12$ & $121.7(4)$ \\
\hline $\mathrm{C} 3-\mathrm{C} 2-\mathrm{C} 1$ & $122.6(4)$ & $\mathrm{C} 8-\mathrm{C} 13-\mathrm{C} 9$ & $130.2(4)$ \\
\hline $\mathrm{C} 3-\mathrm{C} 2-\mathrm{S} 2$ & $117.4(4)$ & $\mathrm{C} 12-\mathrm{C} 13-\mathrm{C} 9$ & $107.9(4)$ \\
\hline $\mathrm{C} 1-\mathrm{C} 2-\mathrm{S} 2$ & $120.0(4)$ & $\mathrm{C} 9-\mathrm{C} 14-\mathrm{C} 15$ & $123.4(4)$ \\
\hline $\mathrm{C} 2-\mathrm{C} 3-\mathrm{C} 4$ & $118.2(5)$ & $\mathrm{C} 9-\mathrm{C} 14-\mathrm{C} 16$ & $122.2(4)$ \\
\hline $\mathrm{C} 2-\mathrm{C} 3-\mathrm{H} 3$ & 120.8 & $\mathrm{C} 15-\mathrm{C} 14-\mathrm{C} 16$ & $114.4(4)$ \\
\hline $\mathrm{C} 4-\mathrm{C} 3-\mathrm{H} 3$ & 121.0 & $\mathrm{~N} 15-\mathrm{C} 15-\mathrm{C} 14$ & $177.0(6)$ \\
\hline $\mathrm{C} 11-\mathrm{C} 4-\mathrm{C} 3$ & $121.7(4)$ & $\mathrm{N} 16-\mathrm{C} 16-\mathrm{C} 14$ & $178.2(6)$ \\
\hline $\mathrm{C} 11-\mathrm{C} 4-\mathrm{N} 4$ & $122.2(4)$ & $\mathrm{S} 2-\mathrm{N} 2-\mathrm{H} 21$ & 110.1 \\
\hline $\mathrm{C} 3-\mathrm{C} 4-\mathrm{N} 4$ & $115.8(4)$ & $\mathrm{S} 2-\mathrm{N} 2-\mathrm{H} 22$ & 114.0 \\
\hline $\mathrm{C} 12-\mathrm{C} 5-\mathrm{C} 6$ & $122.6(4)$ & $\mathrm{H} 21-\mathrm{N} 2-\mathrm{H} 22$ & 111.9 \\
\hline $\mathrm{C} 12-\mathrm{C} 5-\mathrm{N} 5$ & $122.7(4)$ & $\mathrm{O} 41-\mathrm{N} 4-\mathrm{O} 42$ & $125.1(4)$ \\
\hline $\mathrm{C} 6-\mathrm{C} 5-\mathrm{N} 5$ & $114.5(4)$ & $\mathrm{O} 41-\mathrm{N} 4-\mathrm{C} 4$ & $118.5(4)$ \\
\hline $\mathrm{C} 7-\mathrm{C} 6-\mathrm{C} 5$ & $118.5(4)$ & $\mathrm{O} 42-\mathrm{N} 4-\mathrm{C} 4$ & $116.3(4)$ \\
\hline $\mathrm{C} 7-\mathrm{C} 6-\mathrm{H} 6$ & 120.6 & $\mathrm{O} 52-\mathrm{N} 5-\mathrm{O} 51$ & $125.0(4)$ \\
\hline $\mathrm{C} 5-\mathrm{C} 6-\mathrm{H} 6$ & 120.8 & $\mathrm{O} 52-\mathrm{N} 5-\mathrm{C} 5$ & $117.0(4)$ \\
\hline $\mathrm{C} 6-\mathrm{C} 7-\mathrm{C} 8$ & $121.9(4)$ & $\mathrm{O} 51-\mathrm{N} 5-\mathrm{C} 5$ & $117.9(4)$ \\
\hline $\mathrm{C} 6-\mathrm{C} 7-\mathrm{S} 7$ & $117.8(3)$ & S7-N7- & 103.2 \\
\hline $\mathrm{C} 8-\mathrm{C} 7-\mathrm{S} 7$ & $120.2(4)$ & $\mathrm{S} 7-\mathrm{N} 7-\mathrm{H} 72$ & 107.3 \\
\hline $\mathrm{C} 13-\mathrm{C} 8-\mathrm{C} 7$ & $118.3(4)$ & $\mathrm{H} 71-\mathrm{N} 7-\mathrm{H} 72$ & 124.7 \\
\hline $\mathrm{C} 1-\mathrm{C} 2-\mathrm{S} 2-\mathrm{N} 2$ & $79.0(4)$ & $\mathrm{C} 8-\mathrm{C} 7-\mathrm{S} 7-\mathrm{N} 7$ & $96.3(4)$ \\
\hline
\end{tabular}

Hydrogen-bond geometry $\left(\AA,{ }^{\circ}\right)$

\begin{tabular}{lllll}
\hline$D-\mathrm{H} \cdots A$ & $D-\mathrm{H}$ & $\mathrm{H} \cdots A$ & $D \cdots A$ & $D-\mathrm{H} \cdots A$ \\
\hline $\mathrm{N} 2-\mathrm{H} 21 \cdots \mathrm{O} 72^{\mathrm{i}}$ & 0.89 & 2.03 & $2.878(6)$ & 159 \\
$\mathrm{~N} 2-\mathrm{H} 22 \cdots \mathrm{O} 52^{\mathrm{ii}}$ & 0.89 & 2.10 & $2.925(5)$ & 153 \\
$\mathrm{~N} 7-\mathrm{H} 71 \cdots \mathrm{O} 41^{\mathrm{iii}}$ & 0.89 & 2.51 & $3.116(6)$ & 126 \\
$\mathrm{~N} 7-\mathrm{H} 72 \cdots \mathrm{N} 2^{\mathrm{iv}}$ & 0.89 & 2.24 & $3.103(6)$ & 162
\end{tabular}

Symmetry codes: (i) $-x+3 / 2, y-1 / 2, z+1 / 2$; (ii) $x, y-1, z$; (iii) $-x+1,-y+2, z-1 / 2$; (iv) $-x+1,-y+1, z-1 / 2$. 